\title{
Suppression of choroidal neovascularization by Endostar in rats
}

\author{
JIAN LIU, PANPAN YE, ZHAOAN SU, KANA LIN, FENGYING HE and WEN XU \\ Eye Center, Second Affiliated Hospital, Zhejiang University, Hangzhou, Zhejiang 310009, P.R. China \\ Received July 26, 2013; Accepted April 14, 2014
}

DOI: $10.3892 / \mathrm{mmr} .2014 .3132$

\begin{abstract}
Choroidal neovascularization (CNV) is common in various retinal and choroidal diseases, and may result in severe and irreversible loss of vision. Our previous studies suggested that Endostar, a novel recombinant endostatin, is able to inhibit the proliferation and migration of choroid-retinal endothelial cells. To further evaluate the effect of Endostar on the formation of $\mathrm{CNV}$ in vivo, a rat model of laser-induced $\mathrm{CNV}$ was constructed and Endostar or phosphate-buffered saline treatment was administered intravitreally every other day. Using fluorescein angiography (FA), reduced CNV incidence and leakage grade was observed in the Endostar group. In addition, $\mathrm{CNV}$ area and maximal thickness were prominently reduced in the Endostar group measured by choroid flat mounts and sections. Furthermore, vascular endothelial growth factor (VEGF), hypoxia-inducible factor $1 \alpha$ and chemokine C-X-C motif ligand 1 were markedly reduced in the Endostar group as determined by quantitative polymerase chain reaction and downregulation of VEGF was also verified by western blot analysis at the protein level. This study demonstrates that Endostar suppressed CNV in a rat model, which may be largely mediated by the downregulation of VEGF and other angiogenic molecules.
\end{abstract}

\section{Introduction}

Choroidal neovascularization (CNV) refers to the abnormal growth of choroidal vessels, which extend through Bruch's membrane into the sub-retinal pigment epithelium (RPE) or subretinal space (1). Its formation is widely considered as the outcome of breaking the balance between angiogenic and angiostatic factors. Among these factors, vascular endothelial growth factor (VEGF), one of numerous pro-angiogenic molecules, has been indicated as a critical factor in CNV growth in a number of experimental and clinical studies (2-4).

Correspondence to: Dr Wen Xu, Eye Center, Second Affiliated Hospital, Zhejiang University, 88 Jiefang Road, Hangzhou, Zhejiang 310009, P.R. China

E-mail: xuwenhz2002@aliyun.com

Key words: choroidal neovascularization, vascular endothelial growth factor, drug therapy
Endostar is a novel recombinant human endostatin, which was approved by the State Food and Drug Administration of China for the treatment of non-small-cell lung cancer in 2005 (5). It has a six-histidine tag at the N-terminal of the protein, which may simplify purification and improve the stability of the protein (6). Previous studies have indicated that Endostar reduces micro blood vessel density and lymphatic micro vessel density to control tumor growth in vivo via the downregulation of VEGF-A and VEGF-C (7).

Considering the potential effect of endostatin on experimental CNV $(8,9)$, Endostar was administered to laser-induced $\mathrm{CNV}$ in rats to determine whether this drug may prevent $\mathrm{CNV}$ formation. In addition, the potential underlying mechanisms were investigated.

\section{Materials and methods}

Animals. Male brown Norway rats (age, 8 weeks) obtained from the Animal Supply Center of Zhejiang Academy of Medical Science (Hangzhou, China) were used in accordance with the Association for Research in Vision and Ophthalmology Statement for the Use of Animals in Ophthalmic and Vision Research. The present study was approved by the Ethical Committee of the School of Medicine, Zhenjiang University (Hangzhou, China). Anesthesia was induced by intraperitoneal injection of $1 \%$ sodium pentobarbital $(45 \mathrm{mg} / \mathrm{kg}$ body weight), and then the ocular surface was anesthetized with topical instillation of oxybuprocaine hydrochloride. The pupils were dilated with topical $1 \%$ tropicamide (Santen Pharmaceutical Co., Ltd., Osaka, Japan).

Induction of $C N V$ and administration of Endostar or vehicle. Eight burns of 532-nm diode laser photocoagulation $(50 \mu \mathrm{m}$, $0.05 \mathrm{sec}, 300 \mathrm{~mW}$ ) were delivered to each retina with the slit lamp delivery system of a diode laser (OcuLight GL; Iridex, Mountain View, CA, USA) to induce the CNV model. The laser spots were applied between the major retinal vessels 2-4 disc diameters from the optical disc. Ocular contact lenses (Ocular Instruments Inc., Bellevue, WA, USA) filled with one drop of methylcellulose were applied to the rat cornea. Production of a bubble at the time of laser exposure was confirmed for each spot. A total of 40 rats received laser photocoagulation treatment in each eye and were then divided equally into two groups, the Endostar group and phosphate-buffered saline (PBS) control group, at random. Each rat received an intravitreal injection of $10 \mu \mathrm{l}$ Endostar (5 mg/ml; Yantai Sincere Machinery Co., Ltd., Yantai, 
China) or $10 \mu$ l PBS bilaterally using a sterile $10 \mu$ 1 Hamilton syringe (Hamilton Co., Reno, NV, USA) with a 33G needle following laser photocoagulation every other day for two weeks. This surgery was completed under visualization of Zeiss OPMI 6SFR operating microscope (Zeiss Humphrey Systems, Dublin, CA, USA). All examinations of the rats were performed 14 days following the photocoagulation.

Fluoresceinangiogram $(F A)$. The activity ofCNV was evaluated by assessment of late-phase FA, captured 8-10 min following intraperitoneal injection of $0.1 \mathrm{ml}$ of $10 \%$ fluorescein sodium (Alcon, Fort Worth, TX, USA) at day 14 in the rat models. The anesthetized rats with dilated pupils were observed using a digital imaging system (Heidelberg Retina Angiograph II; Heidelberg Engineering, Heidelberg, Germany). Leakage was defined as the presence of a hyperfluorescent spot that increased in size with time. Angiograms were graded by a previously established scheme (10) as follows: 0, no leakage; 1, slight leakage (hyperfluorescent lesion without progressive increase in size or intensity); 2, moderate leakage (hyperfluorescence increasing in intensity but not in size); 3 , prominent leakage (hyperfluorescence increasing in intensity and in size). In order to ensure the effectiveness and accuracy of the evaluation, the angiograms were assessed by two independent graders, who were blinded to the intervention.

Choroidalflat mounts. CNV area was measured with choroidal flat mounts in accordance with the previously reported methods (11). The rats were anesthetized and perfused through the left ventricle with $50 \mathrm{ml}$ vehicle, followed by $20 \mathrm{ml}$ of $5 \mathrm{mg} / \mathrm{ml}$ FITC-dextran (MW, 2x10 ${ }^{6}$; Sigma, St. Louis, MO, USA) in $10 \%$ (wt/vol) gelatin. The eyes were enucleated and fixed in $4 \%$ paraformaldehyde for $1 \mathrm{~h}$. Next, the anterior segment and retina were removed from the eyecup. Four to six relaxing radial incisions were made, and the remaining RPE-choroid-sclera complex was coverslipped. Images of the choroidal flat mounts were then captured under an epifluorescent microscope (Leica Microsystems, Wetzlar, Germany). For measuring the CNV lesion, the green hyperfluorescent area at the laser spot in the flat mounts was measured by Image-Pro Plus software (Media Cybernetics, Bethesda, MD, USA).

Immunofluorescent staining. Enucleated eyes from rats were immediately fixed in $4 \%$ paraformaldehyde at $4^{\circ} \mathrm{C}$ for $12 \mathrm{~h}$. After the anterior segment and lens were removed, the remaining eyecup was cytoprotected with $30 \%$ sucrose and then embedded in Tissue-Tek OCT compound (Sakura Finetech, Tokyo, Japan). The eyecups were cut into $7-\mu \mathrm{m}$ sections. Following blocking with $5 \%$ bovine serum albumin (BSA) for $1 \mathrm{~h}$, these cryo-sections were incubated with the primary rabbit anti-CD31 monoclonal antibody (Abcam, Cambridge, $\mathrm{UK}$ ) at $4^{\circ} \mathrm{C}$ overnight. The sections were then incubated with secondary antibody, FITC-conjugated goat-anti-rabbit IgG F(ab)2 fragment (Santa Cruz Biotechnology, Santa Cruz, CA, USA) for $1 \mathrm{~h}$. The slides were viewed with an epifluorescent microscope (Leica Microsystems). CNV thickness was then measured vertically from the adjacent RPE layer to the top of the CNV. Consecutive sections were examined to select maximal CNV thickness. The thickness was measured by Image-Pro Plus software (Media Cybernetics).
Quantitative polymerase chain reaction ( $q P C R)$. Total RNA was extracted from six rat eyecups in each group using TRIzol reagent and the cDNA was generated using the SuperScript III First-Strand Synthesis system (both from Invitrogen Life Technologies, Carlsbad, CA, USA). qPCR was performed using an ABI Prism 7500 ${ }^{\mathrm{TM}}$ instrument (Applied Biosystems, Carlsbad, CA, USA) and the SYBR Premix Dimer Eraser (Takara Bio, Inc., Dalian, China) according to the manufacturer's instructions. The mRNA expression of VEGF, hypoxia-inducible factor $1 \alpha(\mathrm{HIF}-1 \alpha)$, chemokine $\mathrm{C}-\mathrm{X}-\mathrm{C}$ motif ligand 1 (CXCL1), angiogenin (Ang), fms-related tyrosine kinase 1 (Flt-1) and pigment epithelium-derived factor (PEDF) were analyzed after normalization against the expression of GAPDH. Primer sequences were as follows: Forward: 5'-AGGCAGCTTGAGTTAAACGAACGTA-3' and reverse: 5'-AGGTCTAGTTCCCGACCCTGA-3' for VEGF; forward: 5'-TCTAGTGAACAGGATGGAATGGAG-3' and reverse: 5'-TCGTAACTGGGCTGTGGTAA-3' for HIF-1 $\alpha$; forward: 5'-TGCACCCAAACCGTC-3' and reverse: 5'-ACGCCA TCGGTGCAATCTA-3' for CXCL1; forward: 5'-CCAGTT GCAAGCATA-3' and reverse: 5'-AGCCATTCTCACA GGCAATAACAAC-3' for Ang; forward: 5'-GAGTGCAAA TGGATGAAG-3' and reverse: 5'-CAGCAATCCATG ATTTGGTA-3' for Flt-1; forward: 5'-GAGTGCCTC CAGAATTGTGTTTGA-3' and reverse: 5'-CCTGCACCCAGT TGTTAATCTCC-3' for PEDF; and forward: 5-CAAGTTCAA CACAGTCA-3' and reverse: 5'-CCATTTGATGTTAG CGGGAT-3' for GAPDH. The PCR conditions for various genes were as follows: 40 cycles of $95^{\circ} \mathrm{C}$ for $2 \mathrm{~min} ; 95^{\circ} \mathrm{C}$ for $5 \mathrm{sec}, 55^{\circ} \mathrm{C}$ for $30 \mathrm{sec}$ and $72^{\circ} \mathrm{C}$ for $34 \mathrm{sec}$.

Western blot analysis. The protein extracts were prepared from three eyecups in each group. The samples were then assessed for protein concentration (Bradford assay; Bio-Rad Laboratories, Munich, Germany). Electrophoresis of proteins was performed with $10 \%$ SDS-polyacrylamide gels. A total of $30 \mu \mathrm{g}$ of protein were loaded on each lane. The protein was electrotransferred to a polyvinylidene difluoride membrane (Millipore, Bedford, MA, USA), which was blocked in Tris-buffered saline with Tween-20 (TBST) containing 5\% (wt/vol) BSA for $1 \mathrm{~h}$ at room temperature and then probed overnight at $4{ }^{\circ} \mathrm{C}$ with rabbit polyclonal anti-VEGF (1:1,000; Santa Cruz Biotechnology, Inc.) or rabbit polyclonal anti- $\beta$-actin antibody $(1: 2,000$; Affinity Bioreagents, Golden, CO, USA). The blots were washed three times with TBST for 15 min per wash and then incubated with anti-rabbit horseradish peroxidase-conjugated IgG secondary antibody (1:1,000; Santa Cruz Biotechnology, Inc.) for $1 \mathrm{~h}$ at room temperature. They were then washed again three times each for $5 \mathrm{~min}$. Chemiluminescent detection was accomplished using the enhanced chemiluminescence kit (Amersham Biosciences, Little Chalfont, UK) and ChemiDoc XRS apparatus (Bio-Rad Laboratories, Hercules, CA, USA). The band intensity was measured using densitometry. The results were expressed as the density ratio relative to $\beta$-actin.

Statistical analysis. The data are expressed as the mean \pm standard error of the mean. Student's t-test was used for statistical comparison of the means between the groups. The changes of leakage of CNV were analyzed using Pearson's $\chi^{2}$ test or Mann-Whitney U test, where appropriate. Statistical analyses 


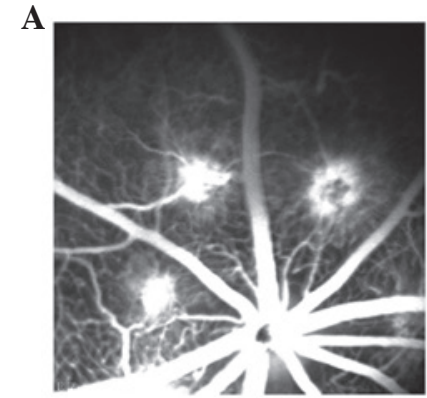

PBS

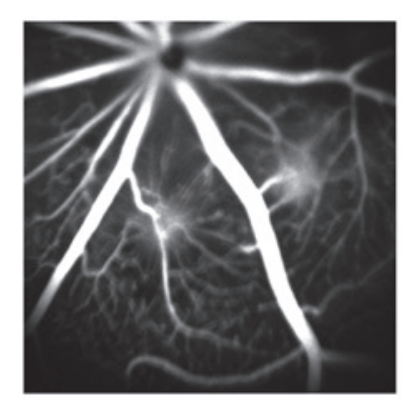

Endostar

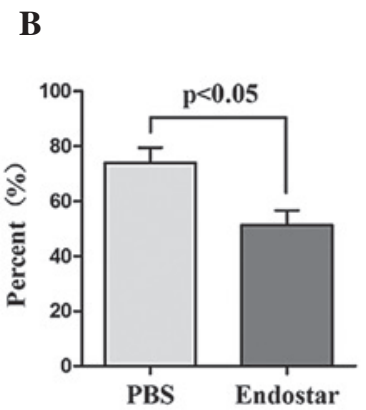

C

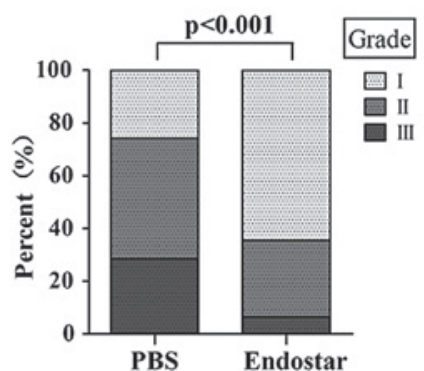

Figure 1. Angiographic analysis of CNV leakage. (A) Fluorescein angiography was performed 14 days following laser photocoagulation and was compared between the PBS and Endostar groups. (B) The incidence and (C) leakage grades of CNV in the Endostar and PBS groups were compared by Pearson's $\chi^{2}$ test and Mann-Whitney U test, respectively. CNV, choroidal neovascularization; PBS, phosphate-buffered saline.

A
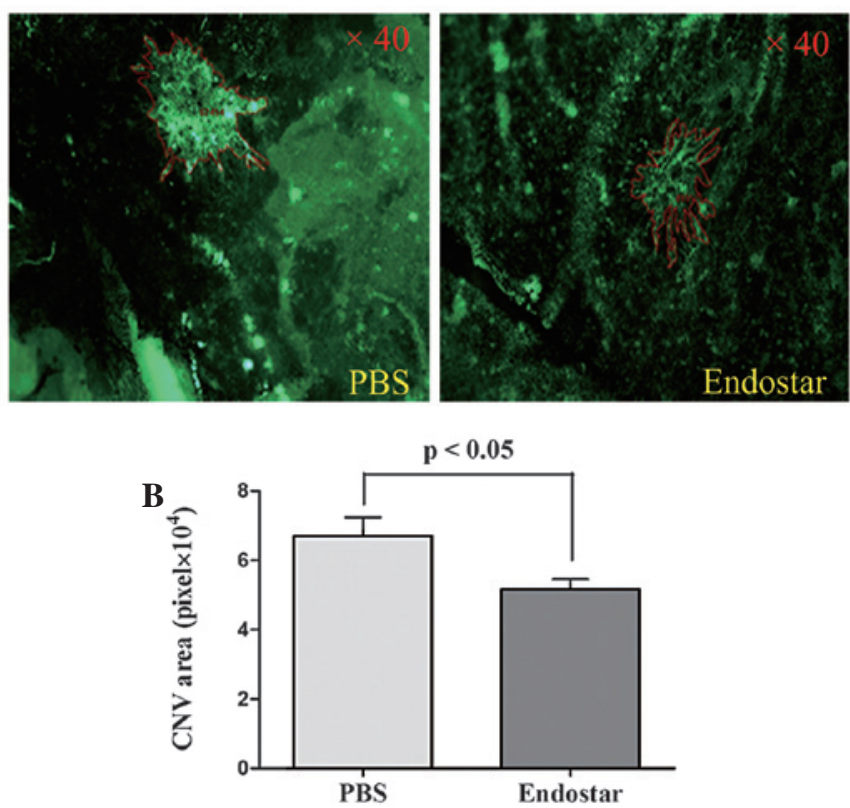

Figure 2. Effect of Endostar on CNV size. CNV area was measured by chorioldal flat mounts at day 14. (A) Representative images are demonstrated in the upper panels, magnification, $\mathrm{x} 40$. (B) The statistic analysis of data is illustrated in the lower panel, the Endostar group (51711 \pm 2880 pixel) vs. the PBS group (67019 \pm 5407 pixel; $\mathrm{P}<0.05$, by Student's t-test). $\mathrm{CNV}$, choroidal neovascularization; PBS, phosphate-buffered saline.

were performed using SPSS software version 16.0 (SPSS, Inc., Chicago, IL, USA). All P-values are two-sided, and $\mathrm{P}<0.05$ was considered to indicate a statistically significant difference.

\section{Results}

Endostar suppresses the activity and volume of $C N V$. The FA in the two groups was compared at day 14 following treatment. The outcome revealed that Endostar-treated rats had reduced CNV incidence and leakage grade compared with the control. CNV was observed in $50.3 \%$ laser spots in Endostar-treated rats ( $n=161$ spots), while it was observed in a significantly higher proportion in control rats $(75.8 \%, \mathrm{n}=153$ spots; $\mathrm{P}<0.05$, by Pearson's $\chi^{2}$ test). The extent of CNV leakage also reduced markedly in Endostar-treated rats $(\mathrm{P}<0.001$, by Mann-Whitney U test; Fig. 1).
A
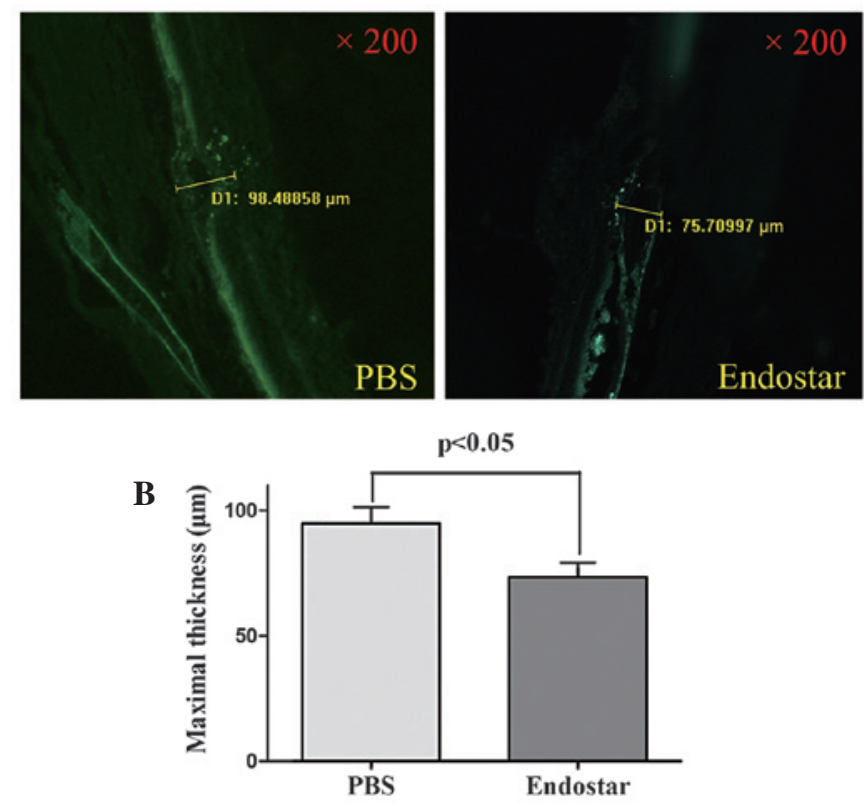

Figure 3.EffectofEndostaronmaximalCNVthickness.(A)Immunofluorescent staining with anti-CD31 antibody for consecutive CNV sections was performed at day 14 to measure the maximal CNV thickness, magnificantion, $\mathrm{x} 200$. (B) The results demonstrated significantly-reduced maximal thickness of CNV in the Endostar group $(73.5 \pm 5.6 \mu \mathrm{m})$ compared with the PBS group $(94.8 \pm 6.4 \mu \mathrm{m} ; \mathrm{P}<0.05$, by Student's t-test). CNV, choroidal neovascularization; PBS, phosphate-buffered saline.

CNV area and the maximal thickness of each CNV were quantified by analysis of fluorescence on day 14 following Endostar or PBS treatment. The mean CNV area in Endostar-treated rats ( $\mathrm{n}=39$ spots) was significantly less than that in PBS-treated rats ( $n=42$ spots; $51711 \pm 2880$ vs. $67019 \pm 5407$ pixel, $\mathrm{P}<0.05$, by Student's t-test; Fig. 2). Furthermore, the maximal thickness of CNV in the Endostar group was also reduced significantly compared with the PBS group (73.5 $\pm 5.6 \mu \mathrm{m}$ vs. $94.8 \pm 6.4 \mu \mathrm{m}$, respectively; $\mathrm{n}=11$ spots per group, $\mathrm{P}<0.05$, by Student's t-test; Fig. 3).

Endostar downregulates angiogenesis-associated molecules. To determine the effect of Endostar on angiogenesis-associated molecules, eyecups of rat models at day 14 were collected, and qPCR was performed to detect the expression changes 


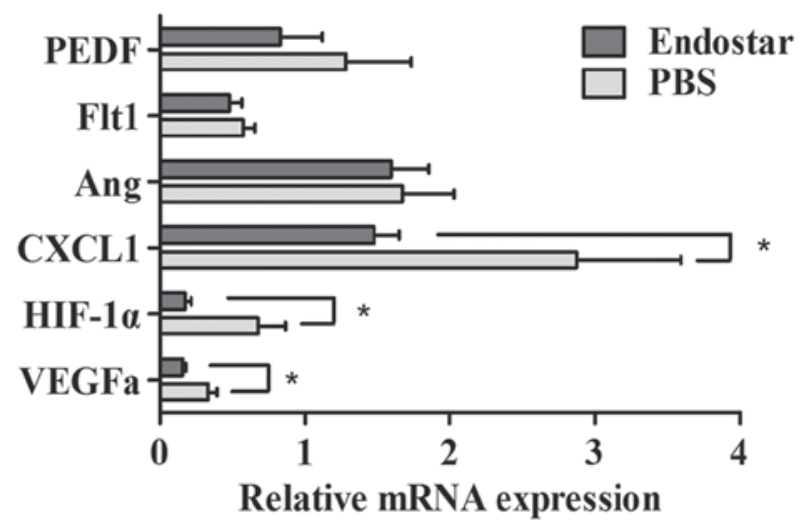

Figure 4. Gene regulation by Endostar in $\mathrm{CNV}$ as determined with qPCR. qPCR demonstrated that angiogenic molecules, VEGF, HIF-1 $\alpha$ and CXCL1, were significantly downregulated in the Endostar group compared with the PBS group ("P $<0.05$, by Student's t-test). CNV, choroidal neovascularization; qPCR, quantitative polymerase chain reaction; PEDF, pigment epithelium-derived factor; Flt1, fms-related tyrosine kinase 1; Ang, angiogenin; HIF-1 $\alpha$, hypoxia-inducible factor $1 \alpha$ VEGF, vascular endothelial growth factor; CXCL1, chemokine C-X-C motif ligand 1; PBS, phosphate-buffered saline.

A

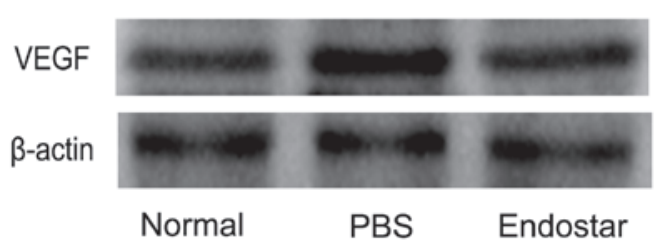

B

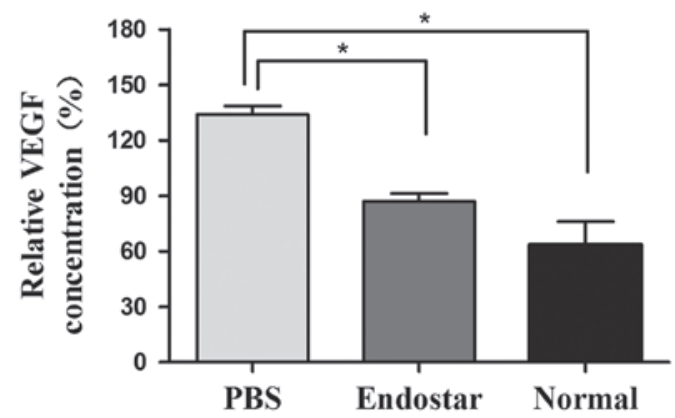

Figure 5. Western blot analysis of VEGF protein expression. (A) The results demonstrated that VEGF was upregulated in laser-induced CNV rats compared with the normal rats. (B) Endostar significantly suppressed the VEGF expression in laser-induced CNV ( $\mathrm{n}=3$ each group; ${ }^{\mathrm{P}}<0.05$, by Student's t-test). CNV, choroidal neovascularization; VEGF, vascular endothelial growth factor; PBS, phosphate-buffered saline.

of certain molecules between the two groups and the results demonstrated that at the mRNA level, VEGF, HIF-1 $\alpha$ and CXCL1 were significantly downregulated in the Endostar group ( $\mathrm{n}=6$ per group; $\mathrm{P}<0.05$, by Student's t-test). However, no significant changes were observed in the expression of Ang, Flt-1 and PEDF (Fig. 4). Furthermore, as verified by western blot analysis, Endostar significantly suppressed the expression of VEGF at the protein level compared with the PBS group $(n=3$ per group; $P<0.05$, by Student's t-test), although the two groups demonstrated upregulated expression of VEGF compared with the control group without laser photocoagulation (Fig. 5).

\section{Discussion}

Endostatin is a broad-spectrum endogenous angiostatic protein, which is proposed to inhibit E-cadherins, matrix metalloproteinases (MMPs), the VEGF signaling pathway, cell survival-related genes, STATs and other key molecules in angiogenesis (12-15). Compared with endostatin, Endostar has an improved function and longer half-life (16). The anti-vescularization properties of Endostar are widely conisdered to be correlated with VEGF $(7,17)$.

In the present study, the angiostatic effects of Endostar were investigated in a laser-induced CNV model. As exhibited by FA, Endostar-treated rats had a significantly reduced incidence of CNV and grade of fluorescence leakage than the PBS group at day 14. To compare the volume of CNV, the choroidal flat mounts and the maximal CNV thickness were detected separately. The results indicated that Endostar treatment significantly decreased the volume of CNV. qPCR and western blot analysis of eyecups in each group confirmed the hypothesis that Endostar exerts its angiostatic effect by regulating the transcription and expression of VEGF, the vital molecule in angiogenesis. In addition, Endostar may also affect other important factors in the angiogenic network. A previous study suggested that RPE cell-derived HIF-1 $\alpha$ is a pivotal contributor to CNV (18). The present results demonstrated an evident decrease in the HIF-1 $\alpha$ mRNA in the Endostar group, which may contribute to CNV suppression. Neutrophil invasion was characteristic of early inflammatory responses during laser-induced $\mathrm{CNV}$, which promoted the early development of CNV possibly via the secretion of angiogenic growth factors (19). CXCR2 is considered to have a crucial role in neutrophil infiltration, while its ligand CXCL1 has potent angiogenic activity (20). Therefore, the downregulation of CXCL1 in the Endostar group may also be conducive to the suppression of CNV.

The present study does however have a number of limitations. Although this CNV model shares histological and angiographic features with the human disease, this acute wound-healing model cannot absolutely reflect the chronic disease found clinically. However, it remains useful to understand the process of CNV development and investigate novel therapeutic agents and strategies. Another limitation of the present study is the absence of further exploration of the appropriate dosage of Endostar for intravitreal injection. Although based on the experience from other experiments, it was possible to observe CNV suppression in the present rat model, further studies are required to examine the efficiency and safety of Endostar and to determine the optimal dosage for future application.

In conclusion, the present study demonstrated that Endostar is able to suppress newly-formed CNV by reducing the expression of VEGF and other angiogenic factors. Therefore, it has potential for clinical CNV treatment alone or as an adjuvant therapy.

\section{Acknowledgements}

This study was supported by the Youth Program of the National Natural Science Foundation of China (grant no. 11104246/A040414), the Zhejiang Natural Science Foundation (grant no. Y2100380), the Zhejiang Science 
and Technology Department Public Project (grant no. 2010C33085), the Zhejiang Key Innovation Team Project (grant no. 2009R50039), the Doctoral Fund of Ministry of Education of China (grant no. 20100101120135) and the Key Lab Fund of Zhejiang Province (grant no. 2011E10006).

\section{References}

1. Grossniklaus HE and Green WR: Choroidal neovascularization Am J Ophthalmol 137: 496-503, 2004.

2. Yu MJ, Shen WY, Lai MC, Constable IJ, Papadimitriou JM and Rakoczy PE: The role of vascular endothelial growth factor (VEGF) in abnormal vascular changes in the adult rat eye. Growth Factors 17: 301-312, 2000.

3. Kwak N, Okamoto N, Wood JM and Campochiaro PA: VEGF is major stimulator in model of choroidal neovascularization. Invest Ophthalmol Vis Sci 41: 3158-3164, 2000.

4. Rosenfeld PJ, Brown DM, Heier JS, et al: Ranibizumab for neovascular age-related macular degeneration. N Engl J Med 355: 1419-1431, 2006.

5. Ling Y, Yang Y, Lu N, et al: Endostar, a novel recombinant human endostatin, exerts antiangiogenic effect via blocking VEGF -induced tyrosine phosphorylation of KDR/Flk-1 of endothelial cells. Biochem Biophys Res Commun 361: 79-84, 2007.

6. Song HF, Liu XW, Zhang HN, et al: Pharmacokinetics of His-tag recombinant human endostatin in Rhesus monkeys. Acta Pharmacol Sin 26: 124-128, 2005.

7. Ma X, Yao Y, Yuan D, et al: Recombinant human endostatin endostar suppresses angiogenesis and lymphangiogenesis of malignant pleural effusion in mice. PLoS One 7: e53449, 2012.

8. Marneros AG, She H, Zambarakji H, et al: Endogenous endostatin inhibits choroidal neovascularization. FASEB J 21 3809-3818, 2007.
9. Mori K, Ando A, Gehlbach P, et al: Inhibition of choroidal neovascularization by intravenous injection of adenoviral vectors expressing secretable endostatin. Am J Pathol 159: 313-320, 2001.

10. Kim C, Yu HG and Sohn J: The anti-angiogenic effect of chlorogenic acid on choroidal neovascularization. Korean J Ophthalmol 24: 163-168, 2010.

11. Honda M, Asai T, Umemoto T, Araki Y, Oku N and Tanaka M: Suppression of choroidal neovascularization by intravitreal injection of liposomal SU5416. Arch Ophthalmol 129: 317-321, 2011.

12. Sudhakar A, Sugimoto H, Yang C, Lively J, Zeisberg M and Kalluri R: Human tumstatin and human endostatin exhibit distinct antiangiogenic activities mediated by alpha $\mathrm{v}$ beta 3 and alpha 5 beta 1 integrins. Proc Natl Acad Sci USA 100: 4766-4771, 2003.

13. Nyberg P, Heikkilä P, Sorsa T, et al: Endostatin inhibits human tongue carcinoma cell invasion and intravasation and blocks the activation of matrix metalloprotease-2, -9, and -13. J Biol Chem 278: 22404-22411, 2003.

14. Rehn M, Veikkola T, Kukk-Valdre E, et al: Interaction of endostatin with integrins implicated in angiogenesis. Proc Natl Acad Sci USA 98: 1024-1029, 2001.

15. Abdollahi A, Hahnfeldt P, Maercker C, et al: Endostatin's antiangiogenic signaling network. Mol Cell 13: 649-663, 2004.

16. Jia $\mathrm{H}$ and Kling J: China offers alternative gateway for experimental drugs. Nat Biotechnol 24: 117-118, 2006.

17. Zhao ZZ, Cao Y, Liu ZS, et al: Effects of recombinant human endostatin on peritoneal angiogenesis in peritoneal dialysis rats. Nephrology (Carlton) 16: 599-606, 2011.

18. Lin M, Hu Y, Chen Y, et al: Impacts of hypoxia-inducible factor-1 knockout in the retinal pigment epithelium on choroidal neovascularization. Invest Ophthalmol Vis Sci 53: 6197-6206, 2012.

19. Zhou J, Pham L, Zhang N, et al: Neutrophils promote experimental choroidal neovascularization. Mol Vis 11: 414-424, 2005

20. Strieter RM, Polverini PJ, Kunkel SL, et al: The functional role of the ELR motif in CXC chemokine-mediated angiogenesis. J Biol Chem 270: 27348-27357, 1995. 